\section{Embolic stroke following cardioversion of atrial fibrillation to sinus rhythm with oral amiodarone therapy}

Sir,

Risk of stroke associated with atrial fibrillation is well recognized..$^{1-3}$ Patients not anticoagulated who undergo elective cardioversion are at risk of developing embolic complications. ${ }^{4}$ Amiodarone is frequently used for treatment of atrial fibrillation and it is known to cause chemical cardioversion. We report a case of disabling stroke after amiodarone-induced cardioversion of atrial fibrillation.

A 66 year old woman with a history of paroxysmal atrial fibrillation was treated with amiodarone as she had symptomatic atrial fibrillation of 5 weeks duration. She was not on prophylactic antiarrhythmic drugs and her previous attacks were infrequent and short lasting. Although she was hypertensive she had not suffered from ischaemic heart disease or transient ischaemic attacks. She was on propranolol $160 \mathrm{mg}$ daily for hypertension.

Clinical examination revealed atrial fibrillation at $120 / \mathrm{min}$ and her blood pressure was $120 / 80 \mathrm{mmHg}$. There was no evidence of cardiomegaly, valvular heart disease or carotid bruits. Her chest X-ray was normal and the electrocardiogram confirmed atrial fibrillation. Eleven days after commencing amiodarone the patient felt her heart rhythm change as she no longer experienced palpitations or difficulty in breathing. Approximately 4 hours later she developed sudden onset numbness and weakness of the right arm and leg. She had evidence of hemiparesis and extensor plantar response on the right side and her pulse was regular at a rate of $66 \mathrm{per}$ min. The electrocardiogram confirmed sinus rhythm. Routine blood tests including thyroid function tests were normal. M-mode echocardiography revealed normal sized left atrium, left ventricle and a normal ejection fraction. Computed tomographic brain scan showed evidence of an area of cerebral infarction in the left parietal lobe. Anticoagulation was commenced 3 weeks later and amiodarone therapy was continued. With intensive rehabilitation she made a gradual but satisfactory recovery.

It is likely that this patient suffered from a thromboembolic stroke following chemical cardioversion due to amiodarone. Although she received treatment for hypertension the sudden onset neurological deficit with no progression coincided well with the change in her heart rhythm. As she had suffered from atrial fibrillation in the past she was well aware of the physical symptoms relating to changes in her heart rhythm. This episode of atrial fibrillation had lasted longer than usual and the reversion to sinus rhythm was influenced by the recent addition of amiodarone. The importance of anticoagulation prior to electrical cardioversion in established atrial fibrillation is well documented. ${ }^{4}$ Paroxysmal atrial fibrillation is reported to be associated with lower risk of stroke, ${ }^{5}$ particularly in patients with normal sized left atrium. ${ }^{6}$ There is a paucity of information as regards the incidence of embolic complications after chemical cardioversion with amiodarone. However, as exemplified by this case report, the possibility of cerebral embolization secondary to chemical cardioversion should be borne in mind and anticoagulation should be considered prior to treatment of atrial fibrillation with amiodarone.

\author{
R.S. Senarath Yapa \\ G.J. Green \\ Department of Medicine, \\ Glan Clwyd Hospital. \\ Rhyl, Clwyd LL18 5UJ, UK.
}

\section{References}

1. Wolf, P.A., Dawber, T.R., Thomas, H.E. Jr et al. Epidemiologic assessment of chronic atrial fibrillation and risk of stroke. The Framingham study. Neurology 1978, 28: 973-977.

2. Wolf, P.A., Kannel, W.B., McGee, D.L. et al. Duration of atrial fibrillation and imminence of stroke. The Framingham study. Stroke 1983, 14: 664-667.

3. Roy, D., Marchand, E., Gagne, P. et al. Usefulness of anticoagulant therapy in the prevention of embolic complications of atrial fibrillation. Am Heart $J$ 1986, 112: 1039-1043.

4. Mancini, G.B. \& Goldberger, A.L. Cardioversion of atrial fibrillation: consideration of embolization, anticoagulation, prophylactic pacemaker and longterm success. Am Heart $J$ 1982, 104: 617-621.

5. Peterson, P. \& Godtfredson, J. Embolic complications in paroxysmal atrial fibrillation. Stroke 1986, 17: 622-626.

6. Caplan, L.R., D'cruz, I., Hier, D.B. et al. Atrial size, atrial fibrillation and stroke. Ann Neurol 1986, 19: 158-161.

\section{Very long term diarrhoea due to Campylobacter jejuni}

Sir,

Campylobacter jejuni is a common cause of acute bacterial diarrhoea in children and adults. Campylobacter enteritis is usually a self limiting disease and only a few cases of mild chronic diarrhoea have been published. We describe a very unusual case of long term diarrhoea due to this pathogen.

A 20 year old white man came to the clinic in June 1985 because of chronic diarrhoea which started in infancy. He had never been investigated or treated before. For more than 17 years he had had several bowel movements a day and sometimes at night. The stools were liquid but never bloody. The clinical examination was absolutely normal. Laboratory tests including blood iron, calcium, $\beta$ carotene, magnesium and immunoglobulins were in the normal range. Bacterial culture of the stools revealed the presence of Campylobacter jejuni. There were no parasites. Proctosigmoidoscopy, double contrast barium enema and barium meal were normal. The treatment was erythromycin $500 \mathrm{mg}$ orally four times a day for 10 days. Diarrhoea disappeared during the treatment and the culture of the stools became negative. The patient was followed during three years and never relapsed.

We believe that our case demonstrates that 17 -years 